\title{
Incremented Alkyl Derivatives Enhance Collision Induced Glycosidic Bond Cleavage in Mass Spectrometry of Disaccharides
}

\author{
Sanford Mendonca \\ Department of Chemistry, Louisiana State University, Baton Rouge, Louisiana, USA \\ Richard B. Cole, Junhua Zhu, and Yang Cai \\ Department of Chemistry, University of New Orleans, New Orleans, Louisiana, USA
}

\begin{abstract}
Alfred D. French and Glenn P. Johnson
United States Department of Agriculture, Southern Regional Research Center, Agricultural Research Service, New Orleans, Louisiana, USA
\end{abstract}

Roger A. Laine

Department of Chemistry and Biological Sciences, Louisiana State University, Baton Rouge, Louisiana, USA

\begin{abstract}
Electrospray ionization and collision induced dissociation on a triple quadrupole mass spectrometer were used to determine the effect of spatial crowding of incremented alkyl groups of two anomeric pairs of peralkylated (methyl to pentyl) disaccharides (maltose/ cellobiose and isomaltose/gentiobiose). Protonated molecules were generated which underwent extensive fragmentation under low energy conditions. For both the $1 \rightarrow 4$ and $1 \rightarrow 6 \alpha$ and $\beta$ isomers, at comparable collision energies the methyl derivative exhibited the least fragmentation followed by ethyl, propyl, butyl, and pentyl. Collision energy is converted to rotational-vibrational modes in competition with bond cleavage, as represented by the slope of product/parent ion $(\mathrm{D} / \mathrm{P})$ ratio versus offset energy. Variable rotational freedom at the glycosidic linkage with incremented alkyl groups is hypothesized to be responsible for this effect. Discrimination of anomeric configuration was also assessed for these stereoiosmeric disaccharides. A systematic study showed that an increasing discrimination was attained for the $1 \rightarrow 4$ isomeric pair as the size of the derivative increased from methyl to pentyl. No anomeric discrimination was attained for the $1 \rightarrow 6$ isomeric pair. Parent and product ion scans confirmed the consistency of fragmentation pathways among derivatives. Chem- $X$ and MM3 molecular modeling programs were used to obtain minimum energy structures and freedom of motion volumes for the permethylated disaccharides. The modeling results correlated with the fragmentation ratios obtained in the mass spectrometer giving strong indication that the collision induced spectra are dependent on the freedom of rotational motion around the glycosidic bond. (J Am Soc Mass Spectrom 2003, 14, 63-78) @ 2003 American Society for Mass Spectrometry
\end{abstract}

\begin{abstract}
C ollision induced dissociation (CID) of carbohydrates has been used to differentiate position of linkage in either the nonreducing end or second glycosidic bond of trisaccharides [1-3]. Methylation of the compounds appeared to increase frangibility [2], therefore we sought to examine an alkyl series, using glucose disaccharide models. Domon and Richter $[4,5]$ showed anomeric differentiation of terminal sugars on acetylated glycosides, therefore we used two anomeric
\end{abstract}

Published online December 11, 2002

Address reprint requests to Dr. R. A. Laine, Department of Biological Sciences, Louisiana State University, 510 Choppin Hall, Baton Rouge, LA 70803, USA. E-mail: rlaine@earthlink.net pairs of glucose disaccharides, namely $1 \rightarrow 4$ (maltose/ cellobiose) and $1 \rightarrow 6$ (isomaltose/gentiobiose). Mass Spectrometry has been used in the determination of monosaccharide type [6-9], sequence [10-14], linkage position $[1-4,15,16]$, and there have been a few studies on anomeric configuration [17-20]. However anomeric configuration differentiation, in micromolar quantities has long been the stronghold of NMR spectroscopy, enzyme degradation, or chromium trioxide degradation. Traditionally mass spectrometry has not been widely used to differentiate stereoisomers. In particular, anomeric configuration has resisted discernment by rapid and sensitive methods.

Low energy ionization techniques like FAB, ESI, and 
MALDI, capable of generating ions from the condensed phase, have enabled extensive studies of middle molecules such as oligosaccharides. FAB and ESI in particular have been used for studies on the potential of differentiation of linkage and anomeric configuration. Positive ion fast atom bombardment (FAB) in conjunction with low energy CID was used by Laine et al. [1-3] for linkage position analysis in both the reducing and nonreducing terminals of linkage isomeric sets of trisaccharides. The key to this approach was noticing a more facile cleavage of rotationally restricted isomers, more limited in dissipation of collision energy into vibronic formats. The approach of the current study was to increment alkyl substitution, attempting to increase the frangibility of the glycosidic acetal bonds. A secondary objective was to examine whether increased frangibility would also increase sensitivity to structural differences.

Oligosaccharides attached to proteins or lipids participate in a wide spectrum of biological roles determined by their primary structures [21, 22]. Increasing reports show carbohydrates exhibiting a variety of important biological functions, such as anti-coagulants, adhesion ligands, immunomodulators, antigenic microbial recognition factors, and inflammatory response addressins, among others [21, 22]. Rapid methods for detailed characterization of their structures have resisted decades of work. The wide variety of positional and anomeric structures makes it possible for saccharides to form as many as $10^{12}$ distinct structures from as few as six different monosaccharide units [23, 24]. Therefore a complete description of carbohydrate structures requires singling out one possibility from a potentially large heterogeneous set of discrete molecular isomers. Problems encountered in their structural elucidation far exceed those encountered with proteins. Complete structural analysis of di- or oligosaccharides requires determination of composition, molecular mass, sugar sequence, branching, ring size, anomeric configuration, and positions of the interglycosidic acetal linkages. There is no extant universal method for complete structural information from nanomoles of glycans, analogous to those for peptides or nucleotides. Methods currently pursued for oligosaccharide analysis include combinations of the following: Enzymatic degradation [25-28], chemical degradation such as acid hydrolysis [29], periodate oxidation [30-32], reduction and methylation [33-38], chromium trioxide oxidation [39] and nuclear magnetic resonance (NMR) [40-43]. NMR requires micromole quantities of pure material for complex 2-D experiments.

Richter et al. [44, 45] assigned the anomeric configuration of nonreducing end glucopyranosyl ions derived from larger oligoglycosides. The study was later extended to anomeric peracetylated glucose-glucose disaccharides $[4,5]$. In these studies, low energy CID was carried out on precursor ions of peracetylated anomers of $1 \rightarrow 2,1 \rightarrow 3,1 \rightarrow 4,1 \rightarrow 6$ linkage type disaccharides that were generated by FAB or DCI. The C-1 carbenium precursor ions were used and partly successful attempts were made to determine both linkage and anomeric configuration. For anomeric configuration differentiation a weak distinction was made based on the relative abundances of the parent and certain daughter ions. In the case of linkage position, a small discrimination was made for the $1 \rightarrow 2,1 \rightarrow 3$, $1 \rightarrow 4$ positions. Differentiation of the same anomers was also carried out in the high energy regime [5]. Again a differentiation was proposed based on ratios of only two ions without consideration given to the ratios of other ions. Khoo and Dell [46] used fast atom bombardment ionization spectra for assigning the anomeric configurations of pyranose sugars in oligosaccharides. Their method was based upon a FAB-MS analysis of deuteroacetylated derivatives before and after oxidation with chromium trioxide which oxidizes the $\beta$ pyranosides to keto esters leaving the $\alpha$ pyranosides largely intact. Therefore, mass spectrometry was not used alone but to differentiate alternate products of a reactive chemical difference. FAB was also used by Leary and coworkers [18] to examine the anomeric configuration in a series of $1 \rightarrow 3,1 \rightarrow 4$ and $1 \rightarrow 6$ glucosyl-glucose disaccharides through the mass-analyzed, ion kinetic energy spectra of the metastable complexes. They reported that the $\alpha$ linked disaccharide complexes consistently displayed a 20-30\% greater kinetic energy release value than the corresponding $\beta$ disaccharide complexes. They also stated that it was difficult to correlate the kinetic energy release (KER) data with structural characteristics of the individual $\mathrm{Co}$ (acac)2/disaccharide complexes without detailed information on the geometry of the disaccharide complex with bis acetyl acetonate. Positive ion FAB-MS/MS data of derivatized xylobiosides showed some distinction between $\alpha$ and $\beta$ configurations in the unimolecular decomposition spectra of the $[\mathrm{M}+\mathrm{H}]^{+}$ions of peracetylated methyl glycosides [47]. The ratio of $\mathrm{m} / \mathrm{z}$ $259 / 475$ was used as a gauge for $\alpha$ and $\beta$ anomers, however this method did not become widely used. The ion at $m / z 259$ corresponded to cleavage of the glycosidic bond while $\mathrm{m} / \mathrm{z} 475$ corresponded to loss of methanol from the parent ion. In comparing of $\beta / \alpha$ values of the $259 / 475$ ratios for the $1 \rightarrow 2,1 \rightarrow 3$, and $1 \rightarrow 6$ anomers, the $\beta$ anomer consistently gave a higher ratio.

Fast atom bombardment was also used together with mass-analyzed ion kinetic energy (MIKE) spectra and collision induced dissociation in both positive and negative ion modes to attempt differentiation of unsaturated and saturated anomeric C glycosides [48, 49]. Negative ion FAB together with metastable ion and collision activation techniques were used to examine anomeric linkages for all glucose-glucose disaccharides [50]. This analysis was made based on the abundance of the monosaccharide ion at $m / z 221$. Differences between the anomers were small and did not always allow unambiguous identification. Negative ion electrospray was used in an attempt to determine directly the anomeric configuration in a complete series of underi- 
vatized glucopyranosyl disaccharides based on "insource" CID in the nozzle-skimmer region rather than tandem mass spectrometric analysis [17]. The discrimination was based on the ratio of $\mathrm{m} / \mathrm{z} 161 / 341$ monosaccharide/disaccharide which increased as the nozzle to skimmer voltage difference was raised. In-source CID was also used to differentiate between the anomeric configuration of the glycosidic bond in disaccharides and linear oligosaccharides labeled with $p$-aminobenzoic ethylester chromophore [19]. While little or no anomeric discrimination was found for the $1 \rightarrow 3$ and $1 \rightarrow 6$ linkage, the $1 \rightarrow 4$ linkage glucose-glucose disaccharide anomers were discerned. Gaucher and Leary [20] reported differentiation of $\alpha$ and $\beta$ bonds of zincdiethylene triamine $\mathrm{N}$-glycoside complexes of four $1 \rightarrow 4$, and $1 \rightarrow 6$ linked disaccharides by ESI-CID in an ion trap. This differentiation was based on the presence of an ion at $m / z 400$ that was specific for the $\beta 1 \rightarrow 4$, and $\beta 1 \rightarrow 6$ disaccharides, absent in the $\alpha$ linked disaccharides. However application of this method to mixture analysis is circumspect. Furthermore, the diagnostic ion found at $m / z 400$ for the employed $\beta$ linked glucoseglucose and mannose-mannose disaccharides may not be universally present. Nilsson and coworkers [51] reported that both the linkage position and anomeric configuration in glucose-containing disaccharide alditols could be estimated by "multivariate" analysis of mass spectrometry data. Their results indicated that the axial glycosidic bond $(\alpha-D)$ is more easily cleaved than the corresponding equatorial bond $(\beta-D)$. Their models were restricted to glucose-containing disaccharides or disaccharides with a nonreducing terminal galactose residue. A theoretical study of deprotonated glucopyranosyl disaccharide fragmentation was carried out where stereochemistry at the 2-position of the nonreducing ring and anomeric configuration could have a significant effect on disaccharide fragmentation [52]. More recently Williams and Young [53] demonstrated that selected pairs of disaccharides i.e., $1 \rightarrow 4$ linked disaccharides could be differentiated by utilization of their cyclic ferrocenyl boronic esters. The results were based on the differential abundance of the ions in the spectra for the $\alpha$ and $\beta$ isomers. However, no rationale was given for the observed differentiation.

Relative to FAB-MS differentiation of the stereochemistry of the glycosidic bond, electrospray offers more efficient ionization as well as lower background (chemical) noise. Derivatization of carbohydrates by permethylation, peracetylation, and reductive amination with an aromatic or an aliphatic amine has been reported to increase mass spectral response. Burlingame et al. [54] and Reinhold et al. [55] agree that chemical derivatization, the usual approach adopted in structural analysis of carbohydrates, enhanced sensitivity in ESI. We have used electrospray in our work because of its above-stated advantages to examine the effect of alkyl derivatization on CID frangibility of a series of peralkylated disaccharides.

In our previous MS/MS studies [1-3] of linkage position discrimination in a set of linkage isomeric neutral or permethylated trisaccharides for sterically crowded linkages, there were higher glycosidic bond cleavage rates when compared with others under collision activation conditions which was rationalized by molecular modeling [1-3]. Later Leary and Smith [18] also proposed the importance of steric factors as related to energy differences in an attempt to explain differences in kinetic energy release values between the $\alpha$ and $\beta$ anomers in a set of metal ion coordinated glucoseglucose disaccharides. Ohashi et al. [56] stated that one of the future challenges would be to obtain steric information by ES-MS, especially in its CID-MS/MS mode which is a focus of our project.

An alkyl series (ranging from methyl to pentyl) of two pairs of glucose-glucose disaccharides maltose cellobiose and isomaltose gentiobiose were synthesized.

\section{Experimental}

\section{Instrumentation}

Electrospray ionization mass spectrometry was performed using a Micromass Quattro II triple quadrupole mass spectrometer (Micromass Inc., Manchester, England). The sample is introduced via direct infusion at a flow rate of $2-3 \mu \mathrm{L} / \mathrm{min}$. The collision cell is an rf-only hexapole that employed argon as target gas at $1 \times 10^{-5}$ torr, kept constant throughout the experiment. The threshold laboratory collision energy is $5 \mathrm{eV}$ for each derivative, which is then incremented by $4,5,6,7,8 \mathrm{eV}$ for the methyl, ethyl, propyl, butyl, and pentyl derivatives, respectively. Incrementation of the collision energy has been calculated in the center of mass frame of reference based on the following equation:

$$
E_{c o m}=E_{l a b}\left[m_{t} / m_{i}+m_{t}\right]
$$

where $\mathrm{E}_{\mathrm{com}}=$ collision energy in the center of mass frame; $\mathrm{E}_{\text {lab }}=$ collision energy in the lab frame; $\mathrm{m}_{\mathrm{i}}=$ mass of ion; $\mathrm{m}_{\mathrm{t}}=$ mass of target. The product ion spectra resulted from the signal averaging of 25 scans.

\section{High-Resolution Measurements}

High resolution nanoelectrospray-MS was performed on all precursor ions prior to low energy CID studies to unambiguously ascertain the molecular ion. Accurate mass measurements were made with an Ion Spec FTICR.

\section{Synthesis}

Using a modified method of Cicanu and Kerek [57] the underivatized disaccharide (5 $\mathrm{mg}, 0.015 \mathrm{mmol}$ ) was dissolved in DMSO (2mL). Powdered sodium hydroxide $(200 \mathrm{mg}, 5 \mathrm{mmol}$ ) was added and the suspension was stirred for 10 minutes. Finally methyl iodide $(0.21$ $\mathrm{g}, 1.4 \mathrm{mmol}$ ) was added and the solution was stirred at 
room temperature for $24 \mathrm{~h}$. The product was extracted with chloroform $(3 \mathrm{~mL})$, washed several times with distilled water $(4 \times 10 \mathrm{ml})$ and dried with sodium sulfate and then at high vacuum. The same reagents were used for preparation of the higher derivatives from ethyl to pentyl with differences in the proportions of alkyl iodide added. For ethylation $500 \mathrm{uL}$ of ethyl iodide was added in $100 \mathrm{uL}(0.19 \mathrm{~g}, 1.25 \mathrm{mmol})$ aliquots over a period of 5 days after which the products were extracted in chloroform as for methylation. For propylation $600 \mathrm{uL}$ of propyl iodide was added in $100 \mathrm{uL}(0.17$ $\mathrm{g}, 1.03 \mathrm{mmol}$ ) increments over a period of 6 days after which the product was extracted. Butylation was carried out over a period of 7 days in $100 \mu \mathrm{L}(0.16 \mathrm{~g}, 0.87$ $\mathrm{mmol}$ ) increments. Preparation of the ethyl, propyl, and butyl derivatives were all carried out at room temperature. The pentyl derivatives were synthesized by first heating the reaction mixture at $55-60{ }^{\circ} \mathrm{C}$ for $24 \mathrm{~h}$ and then for 8 days at room temperature. Two $\mathrm{ml}$ of pentyl iodide was added to the reaction mixture in aliquots of $200 \mu \mathrm{L}(0.3 \mathrm{~g}, 1.5 \mathrm{mmol})$ for 9 days, after which the product was extracted with chloroform.

\section{Computational Studies}

Chem-X (Oxford) and Sybyl (Tripos) was used to sketch and display structures. Molecular mechanics energy surfaces of the permethylated molecules were prepared using MM3-96 [58]. The default $\epsilon$ of 1.5 was used to mimic molecules in vacuum (e.g. in the mass spectrometer). Block Diagonal minimization was applied with energy optimization to the default convergence value (3.6 cal/mol for the native disaccharides). The conformational analysis was carried out in $20^{\circ}$ intervals, with the torsion angles defined as $\phi=$ O5-C1-O1-C4'and $\psi=$ C5'-C4'-O1-C1. In all optimizations, only these angles were constrained and all other geometric properties were relaxed. The maps show the lowest energy calculated irrespective of the starting model at each $\phi, \psi$ point. Unix shell scripts were written to read in the structures from Chem- $X$ for MM3 minimizations. The $\phi, \psi$ maps were plotted with Surfer (Golden software). Contours are plotted out to $10 \mathrm{kcal} / \mathrm{mol}$ above the respective global minima.

\section{Chemicals and Materials}

The native disaccharides maltose, cellobiose, isomaltose, and gentiobiose were purchased from Sigma Chemicals (St. Louis, MO). Methyl iodide, ethyl iodide, propyl iodide, butyl iodide, and pentyl iodide were obtained from Aldrich Chemical Co. (Milwaukee, WI). All materials were used as received without further purification. Solutions for ESI-MS were prepared by mixing $100 \mathrm{uL}$ of the peralkylated disaccharide of $2 \mathrm{mM}$ in methanol with $1-2 \%$ trifluoroacetic acid.

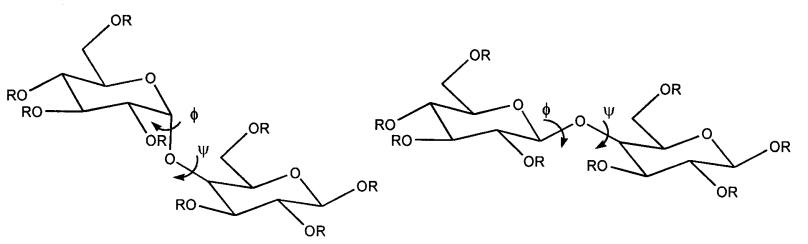

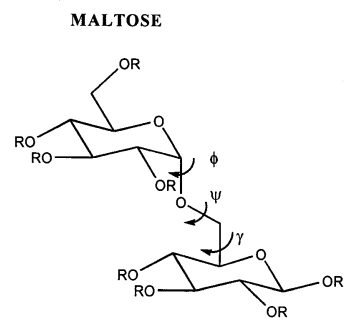

ISOMALTOSE

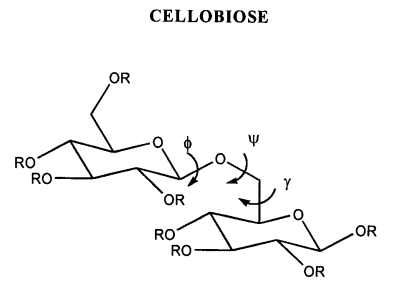

GENTIOBIOSE

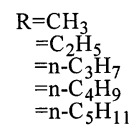

Figure 1. $1 \rightarrow 4$ linked disaccharides (maltose and cellobiose) and $1 \rightarrow 6$ linked disaccharides (isomaltose and gentiobiose).

\section{Results}

Four $\alpha$ and $\beta$ glucosyl-glucose disaccharides, $1 \rightarrow 4$ linked maltose, cellobiose, and $1 \rightarrow 6$ linked isomaltose, gentiobiose derivatized with alkyl groups (methyl to pentyl) (as shown in Figure 1) were analyzed by low energy ESI-MS/MS since this regime allows one to probe subtle variations of ion internal energies. The peralkylated disaccharides preferentially yield intense sodium cationized adducts, which give fragment ions due to both cleavage of the glycosidic bond and sugar ring cleavages and generally require higher energy to fragment $[55,59]$. Protonated molecules of permethylated oligosaccharides had previously been shown to provide facile fragmentation of the glycosidic bond in studies of sugar sequence and branching $[1,2,59]$. The protonated molecules $[\mathrm{MH}]^{+}$were generated in high abundance by addition of 1-2\% trifluoroacetic acid after desalting of the compound with a cation exchange column.

Derivatization of the saccharide in our study with increasing sizes of alkyl substituents provided increases in the steric crowding around the glycosidic bond as shown by molecular modeling. The steric encumbrance on the rotation of the glycosidic bond was incremented by carbon chain length, while keeping constant the number of potential nucleophilic (charge) sites. By increasing the length of the alkyl chain, the basicity and the polarizability of the alkyl groups is increased and the stabilization of the charge on the adjoining ether oxygen is enhanced. Moreover, crowding will restrict rotation of the disaccharide rings causing differences in the manner in which the molecules in the homologous series take up collision energy. 

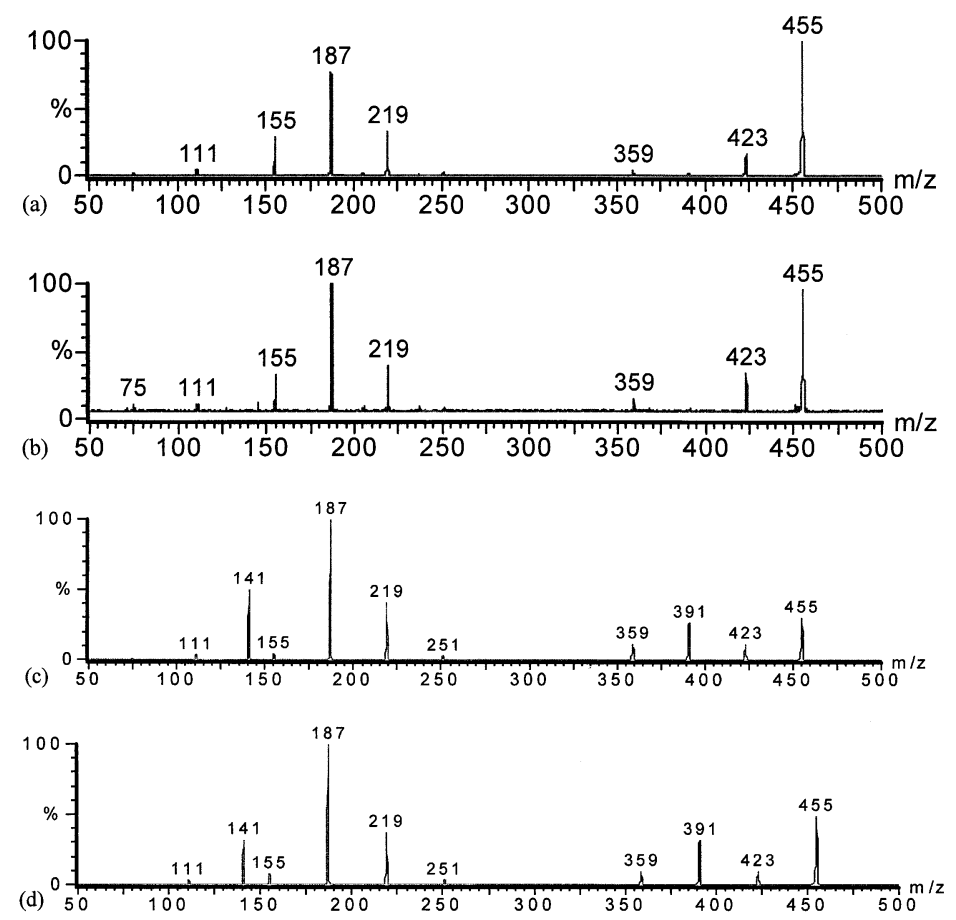

Figure 2. Electrospray tandem mass spectra at $13 \mathrm{eV}$ of permethylated (a) maltose, (b) cellobiose. Electrospray tandem mass spectra at $13 \mathrm{eV}$ of permethylated (c) isomaltose, (d) gentiobiose.

\section{Identity and Mechanistic Pathway for Ion Formation in the Collision Process}

Electrospray tandem mass spectra of permethylated and perpentylated maltose, cellobiose, isomaltose, and gentiobiose at $13 \mathrm{eV}$ (Figure 2a, b, c, d) and $21 \mathrm{eV}$ (Figure $3 a, b, c, d$ ) respectively are illustrated. In separate experiments, high resolution exact mass measurements were performed for each derivative to unambiguously determine the molecular formula of the precursor ion, and to eliminate the possibility of isobaric ions being present. The MS/MS spectrum is characterized by significant fragmentation corresponding to cleavage of the interglycosidic bond. The mechanistic pathway is depicted in Scheme 1. According to the Domon and Costello nomenclature [60] this results in the formation of a B type oxonium ion with retention of the glycosidic oxygen atom by the species formed from the reducing end as shown by Pathway a [61]. The $\mathrm{B}$ ion loses a second methanol (or higher alcohol) via 1,2-elimination to form a diene, an intense $\mathrm{E}$ type ion. The $\mathrm{E}$ ion can undergo a further loss of methanol to form the triene at $m / z 155$, an $E^{\prime}$ type ion. To determine the fragmentation pathway, a daughter ion and parent ion scan was carried out on each product ion of the parent ion at $m / z 455$ (using in this case methylated maltose as an example). It was established that the ions at $m / z 187$ and 155 may be formed via a consecutive methanol loss from $\mathrm{m} / \mathrm{z} 219$. The other monosaccharide ions of importance are ions at $m / z 141$ and 111. The ion at $m / z 111$ is formed by a decomposition of the ion at $\mathrm{m} / \mathrm{z} 187$ by elimination of two methoxy groups through an intermediate at $m / z 141$ (Pathway b). A similar fragmentation pathway was earlier observed by Kochetkov and Chizhov [62]. For the derivatives higher than methyl viz. ethyl, propyl, butyl, and pentyl the ion
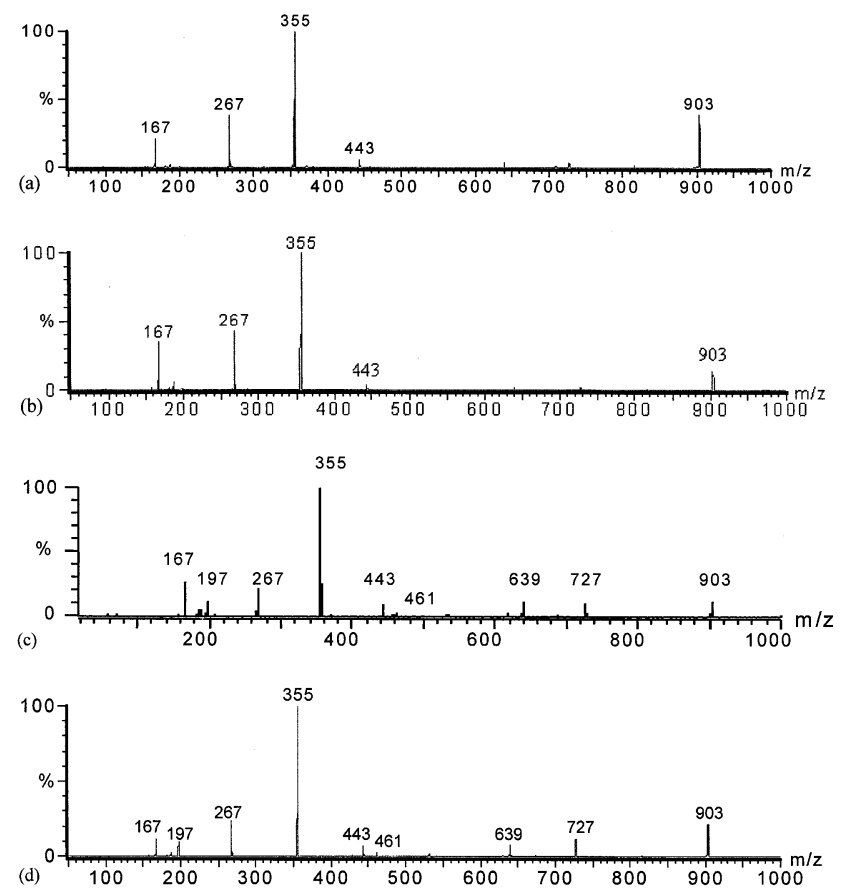

Figure 3. Electrospray tandem mass spectra at $21 \mathrm{eV}$ of perpentylated (a) maltose, (b) cellobiose. Electrospray tandem mass spectra at $21 \mathrm{eV}$ of permethylated (c) isomaltose, (d) gentiobiose. 


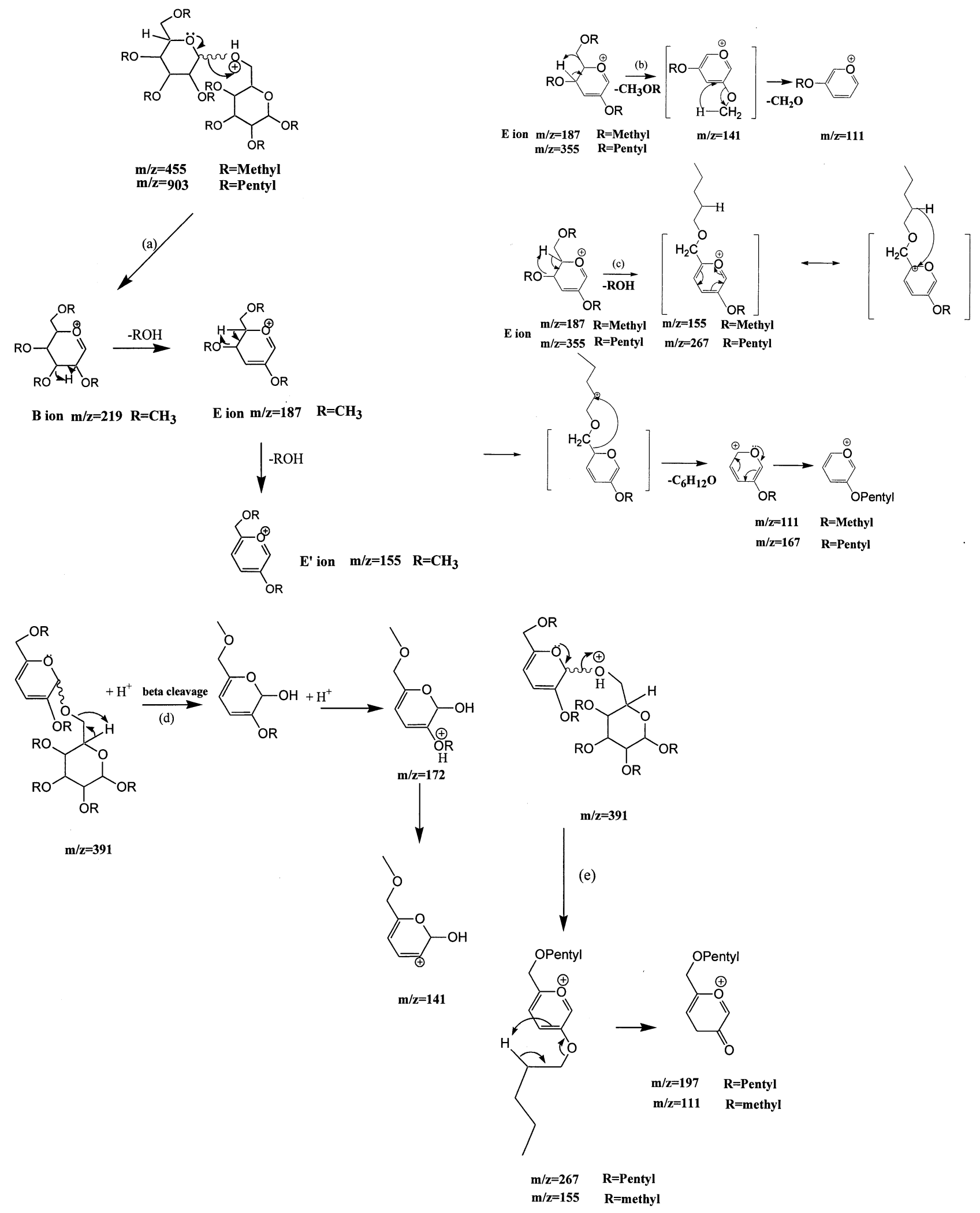

Scheme 1. (a) Ions formed in the electrospray ionization process. Anomeric configuration (wavy line) not specified. (b) and (c) Mechanistic pathway of ions formed in the electrospray ionization process. (d) Mechanistic pathway of ions formed in the electrospray ionization process. (e) Mechanistic pathway of ions formed in the electrospray ionization process. 
Table 1. Comparison of $\Delta \mathrm{E}_{\mathrm{com}}$ and $\Delta \mathrm{E}_{\mathrm{lab}}$ for disaccharide derivatives

\begin{tabular}{lcc}
\hline R Group & $\Delta \mathrm{E}_{\text {Com }}$ & $\Delta \mathrm{E}_{\text {Lab }}$ \\
\hline \hline Methyl & 0.32 & $4 \mathrm{eV}$ \\
Ethyl & 0.33 & $5 \mathrm{eV}$ \\
Propyl & 0.33 & $6 \mathrm{eV}$ \\
Butyl & 0.34 & $7 \mathrm{eV}$ \\
Pentyl & 0.34 & $8 \mathrm{eV}$ \\
\hline
\end{tabular}

analogous to $m / z 111$ (for methyl) could be formed by Pathway b or by a hydride shift (Pathway c). However the methylated derivatives follow only mechanism $b$. The major monosaccharide ion at $\mathrm{m} / \mathrm{z} 141$ is formed by a $\beta$ cleavage of the ion at $m / z 391$ (Pathway d). Dell et al. [63] proposed a similar fragmentation pathway in FAB mass spectrometry. Derivatives higher than methyl could follow a similar pathway (Pathway d) or by elimination of the alkyl chain as an alkene (Pathway e) for alkyl groups higher than methyl. The minor ions at $m / z$ 423, 391, and 359 (Figure 2) are formed by successive losses of methanol (or the corresponding alcohol in the case of the higher derivatives) either by a charge driven or charge remote pathway.

\section{Effect of Increasing Masses of the Parent Ions}

To maintain collision events as isoenergetic, one must compensate for the increasing mass of the precursor ions that change from methyl to pentyl derivatives. This is accomplished by holding the collision energy constant in the center of mass frame of reference [64] (see Experimental section). The collision energy in the center of mass frame of reference $\left(\mathrm{E}_{\mathrm{com}}\right)$ represents the maximum amount of kinetic energy available for conversion into internal energy for an analyte ion of mass $m_{i}$ colliding with a stationary gas atom of mass $m_{t}$. $E_{l a b}$ is incremented with increasing mass of $\mathrm{MH}^{+}$such that the center-of-mass collision energies remain nearly constant. This allows a direct comparison between cleavage rates for the different alkyl derivatives. Compensation for the increase in mass of the target ions is important since only a fraction $\left(E_{\text {com }}\right)$ of $E_{\text {lab }}$ is available for internal excitation [65]. Table 1 shows a comparison of the center-of-mass energy $(\Delta$ Ecom) with the incremented collision energy ( $\Delta$ Elab) for each alkyl derivative. Note that only integer increments of laboratory collision energy are possible on the employed instrument. In this study, anomeric configuration discrimination will be based upon a comparison of the relative rates of competitive and consecutive decompositions. The permethylated derivatives having fewer vibrational and rotational modes would be expected to fragment more completely than the perpentylated derivatives at the same center-of-mass energy on collision with stationary argon. However the glycosidic bond may cleave more easily in the perpentylated derivative as described below. No further correction is made for subtle differences in collision cross-section. Special at-

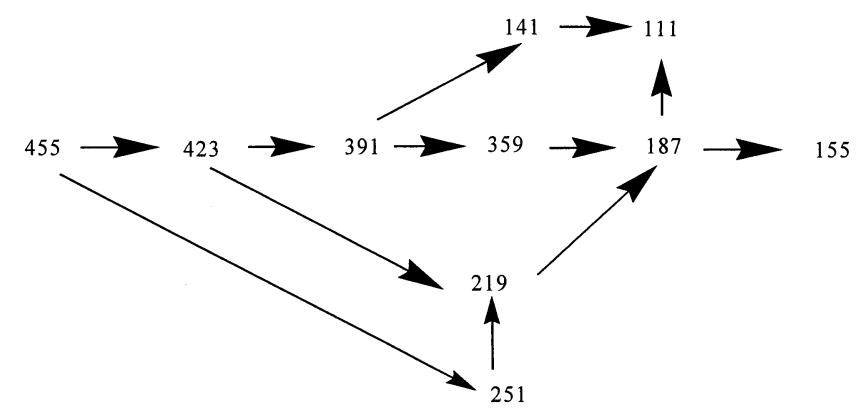

Scheme 2. Fragmentation pathway for permethylated isomaltose.

tention was also paid to the adjustment of the collision gas pressure, such that single or low number collision conditions were maintained in the r.f. only collision hexapole [64]. Maintaining a low constant pressure of $1 \times 10^{-5}$ torr at zero collision offset so that the main ion beam was attenuated by $10 \%$ was used as the criterion for low number collision conditions $[66,67]$. The low energy near single collision conditions employed provides the opportunity to measure subtle differences in dissociation energies.

\section{Methodology Used to Differentiate Stereoisomers}

The MS-MS spectra of each isomer derivatized with the same alkyl group and of the same linkage type show the same parent ion and fragment ions at a certain collision energy. Even though the fragment ions are identical, the abundances of the product ions with respect to parent ion attenuation differ significantly between the two isomers. The MS-MS spectra of decomposition of the $[\mathrm{M}+\mathrm{H}]^{+}$for the four permethylated $(m / z=455)$ and perpentylated $(\mathrm{m} / \mathrm{z}=903)$ glucose-glucose disaccharide isomers $(1 \rightarrow 4$ and $1 \rightarrow 6$ linkage positions) at a collision offset energy of $13 \mathrm{eV}$ and $21 \mathrm{eV}$ (Figures 2 and 3) respectively serve as representative spectra for the entire disaccharide series. The complete fragmentation pathway of all the ions formed in the spectrum of methylated isomaltose is summarized in Scheme 2. This depiction of one possible pathway is deduced by carrying out MS-MS-MS experiments involving sequential dissociation of the product ions (product ion scan) in the spectrum as stated above to obtain their individual product ions. Each alkyl derivative forms homologous sets of ions, with their formation proceeding at different rates. Different anomeric forms always yielded the same product ions but in varying abundances.

In our experiments, the collision energy is varied by incrementing the voltage on a lens before the RF-only quadrupole. Plots of the change in the ion ratio of the combined intensity of relevant daughter ions divided by the intensity of the parent ion against collision offset voltage were constructed (Figure 4 is an example). Plots of the ratio of the daughter to parent ion intensity for each isomer are constructed. To differentiate between $\alpha$ and $\beta$ isomers, a comparison between the absolute 


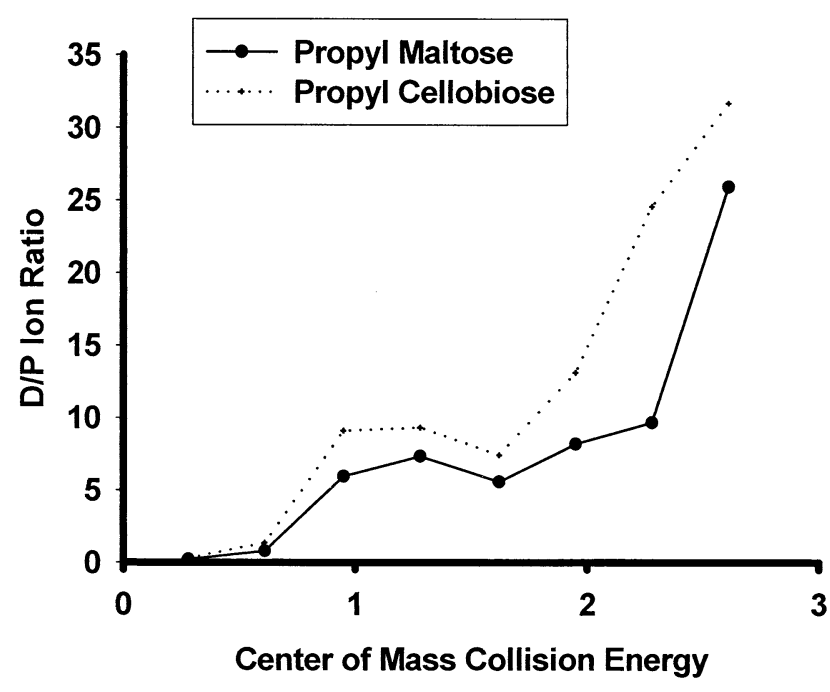

Figure 4. Plot of the collision offset voltage versus daughter/ parent ion ratio.

intensities of the product ions related to cleavage of the glycosidic bond is not as useful as taking the daughter to the parent ratio, which minimizes effects due to variations in instrumental parameters over time. According to the MS-MS-MS spectra (Scheme 2) fragmentation of the glycosidic bond takes place resulting in a series of product ions formed through consecutive decompositions. The daughter ions summed up are $\mathrm{m} / \mathrm{z}$ 219 (the glycosidic bond cleavage ion) and monosaccharide ions at $\mathrm{m} / \mathrm{z} 187,155,141$, and 111. Even though some of the ions are formed by two different pathways and the ion at $\mathrm{m} / \mathrm{z} 187$ in turn fragments to form other ions by two pathways (Scheme 2) they are considered since they are monosaccharide ions which are formed only after cleavage of the glycosidic bond. Fragmentations taking place a number of steps after cleavage of the glycosidic bond (e.g., $\mathrm{m} / \mathrm{z} 141$ and 111) are affected statistically by the strength of that bond. The internal energy acquired by the parent and product ions is related to the structure of the parent ion [68]. Thus, it is reasonable to say that a "memory effect" [69] related to the initial structure of the disaccharide ion is retained in daughter ions formed by consecutive fragmentations. Thus the daughter ion value is obtained by summing up the abundance for all the daughter ions formed once the glycosidic bond is cleaved and the minor disaccharide ions at $\mathrm{m} / \mathrm{z} 423,391$, and 359 are not considered. Incrementing collision energy will enhance the stereochemical differentiation window. This minimizes the necessity to predict in advance the optimum collision energy enabling maximum stereochemical differentiation. The slopes of the daughter to parent ion ratio versus collision energy plots reflect the stability of the glycosidic bond. Information on anomericity is revealed since the $\mathrm{D} / \mathrm{P}$ plots reflect the differences in stability between the two anomers. Furthermore, the collision offset voltage can be better controlled than the pressure of the collision gas and is a more effective way of controlling the internal energy imparted to the molecule.

\section{Effect of the Size of the Alkyl Group on Glycosidic Bond Cleavage}

Figures $5 \mathrm{a}, \mathrm{b}, \mathrm{c}, \mathrm{d}$ are plots showing the daughter to parent ion ratios versus the collision offset voltage for the methylated to pentylated $1 \rightarrow 4$ and $1 \rightarrow 6$ linkage isomers. These plots indicate that the stability of the derivatives decreases in the order of methyl $>$ ethyl $>$ propyl $>$ butyl $>$ pentyl. The larger alkyl disaccharide derivatives dissociate at a higher rate to relieve steric and torsional strain. The allowable number of rotamers is larger upon substitution by smaller alkyl. Dissociation pathways can be described in terms of coupling of molecular vibrations. When the number of rotamers is large, there are a greater number of vibrational states that can couple and dissipate the energy. Even though the number of vibrational modes is increased by an increase in the number of methylenes which can explore more states, pentylated derivatives are unable to dissipate the internal energy as freely as the methylated derivatives among these excess vibrational modes. This is due to the large hindrance in glycosidic bond rotation. Cleavage of the glycosidic bond will therefore be favored because of the incremented predominance of a small number of the total possible conformations of the molecular ion that forces focusing of the available energy on the glycosidic bond. Because of an enhanced rotational barrier, the normal and bend vibrational modes and rotational modes connected to these "frozen-out" rotamers become inaccessible.

Since some of the rotamers are inaccessible for the larger derivatives, the transition state leading to the cleavage products must be more ordered with a reduced entropy of activation. In contrast, a smaller derivative like the permethylated one will have a less ordered transition state with a higher entropy of activation due to the higher number of available conformational states. If the difference in the rotational barrier between permethylated and perpentylated derivatives (presuming they have the same ground state energy) were small enough, it could preclude predominance of kinetic effects. Thus, cleavage of the perpentylated derivative is statistically favored. The permethylated derivatives might also be undergoing an enhanced secondary rotation of the methyl side chain in comparison to the pentyl which further reduces the steric interactions between the methyl side chains during rotation of the glycosidic bond, thus enhancing its freedom of motion. An alternative explanation is that the different derivatives might be lying in different ground state conformations as $\mathrm{R}$ varies. Also, as one goes to the larger alkyl group derivatives there is an increase in the surface area of the molecule enhancing the probability of collision with the target. This in- 

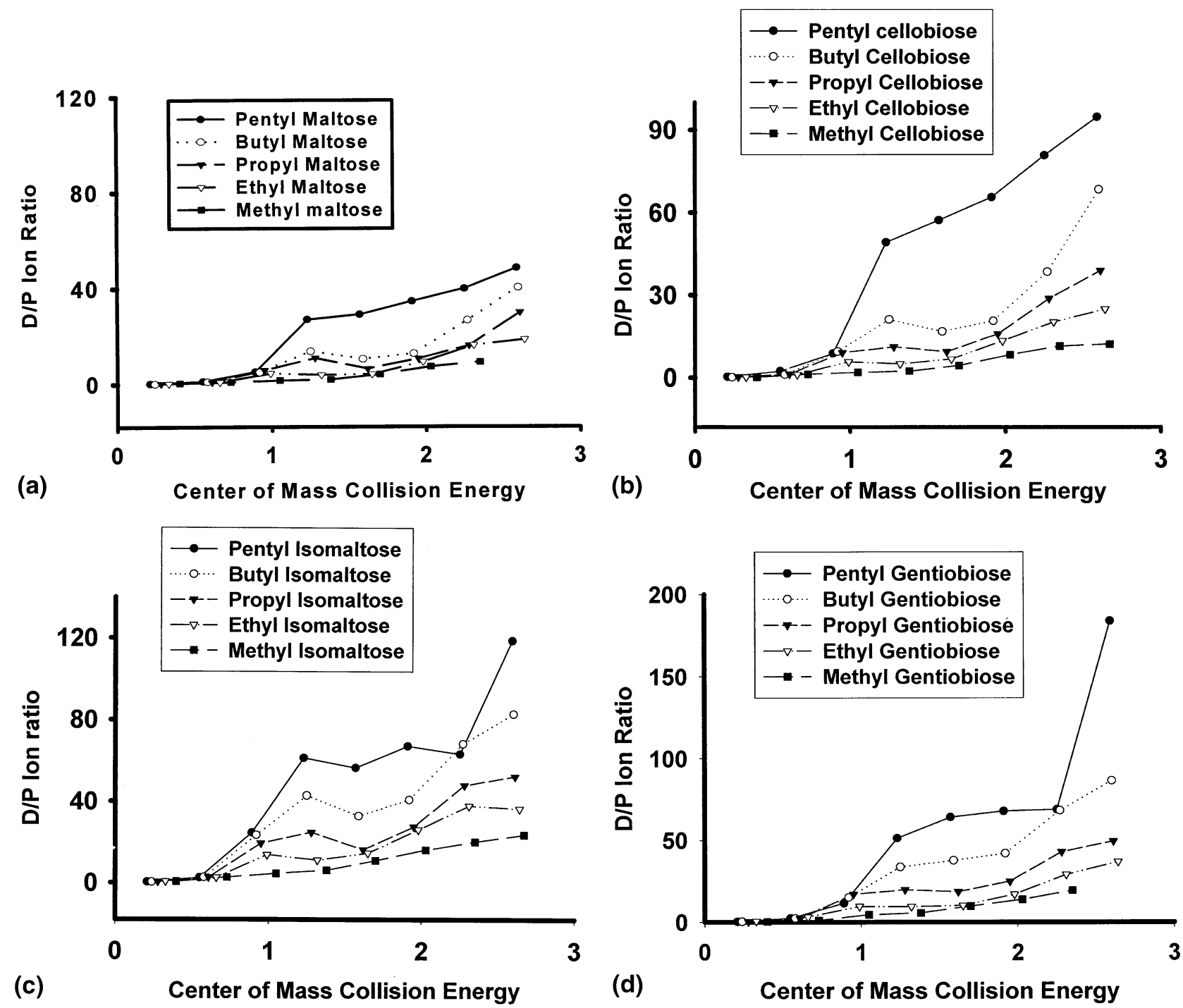

Figure 5. (a) Plot of effect of alkyl groups on glycosidic bond cleavage for $\alpha-(1 \rightarrow 4)$ linked derivatives. (b) Plot of effect of alkyl groups on glycosidic bond cleavage for $\beta-(1 \rightarrow 4)$ linked derivatives. (c) Plot of effect of alkyl groups on glycosidic bond cleavage for $\alpha-(1 \rightarrow 6)$ linked derivatives. (d) Plot of effect of alkyl groups on glycosidic bond cleavage for $\beta-(1 \rightarrow 6)$ linked derivatives.

creased collision cross-section increases the tendency for cleavage of the glycosidic bond.

It is also known that the general trend among alkyl ethers upon protonation is a lengthening of the $\mathrm{O}-\mathrm{CR}$ bond with $\mathrm{R}=$ ethyl to pentyl by $0.14 \mathrm{~A}^{\circ}$ and $\mathrm{R}=$ methyl $0.08 \mathrm{~A}^{\circ}[69]$. This would cause a further increase of intramolecular interaction of alkyl groups already experiencing steric crowding for the larger derivatives, thus augmenting rates of glycosidic cleavage. The gas phase basicities and proton affinities of alkyl ethers are known to decrease with a decrement in size of an alkyl group [70]. More polarizable longer chain alkyl groups will stabilize the positive charge by an inductive effect. The protonated methyl ether group would be more easily eliminated than a corresponding pentyl ether group since the electron donation from the sigma bond attached to the ring would less effectively stabilize the protonated methyl ether. This is manifested by the higher abundances of the disaccharide ions formed for the permethylated derivative by three consecutive losses of methanol in comparison to the perpentylated derivative (Figures 2 and 3).

\section{Anomeric Configuration}

$1 \rightarrow 4$ linkage pairs. Figures $6 \mathrm{a}, \mathrm{b}, \mathrm{c}, \mathrm{d}, \mathrm{e}$ are the plots of the ratio of the daughter to parent ion intensities of the $\beta$ isomer to the $\alpha$ isomer versus the collision offset voltage of the permethylated to perpentylated derivatives for the $1 \rightarrow 4$ linkage compounds. From the five plots, discrimination between the two stereoisomers is made for all pairs of alkylated derivatives, and discernment improves as the size of the alkyl group increments from methyl to pentyl. Low energy collision induced 

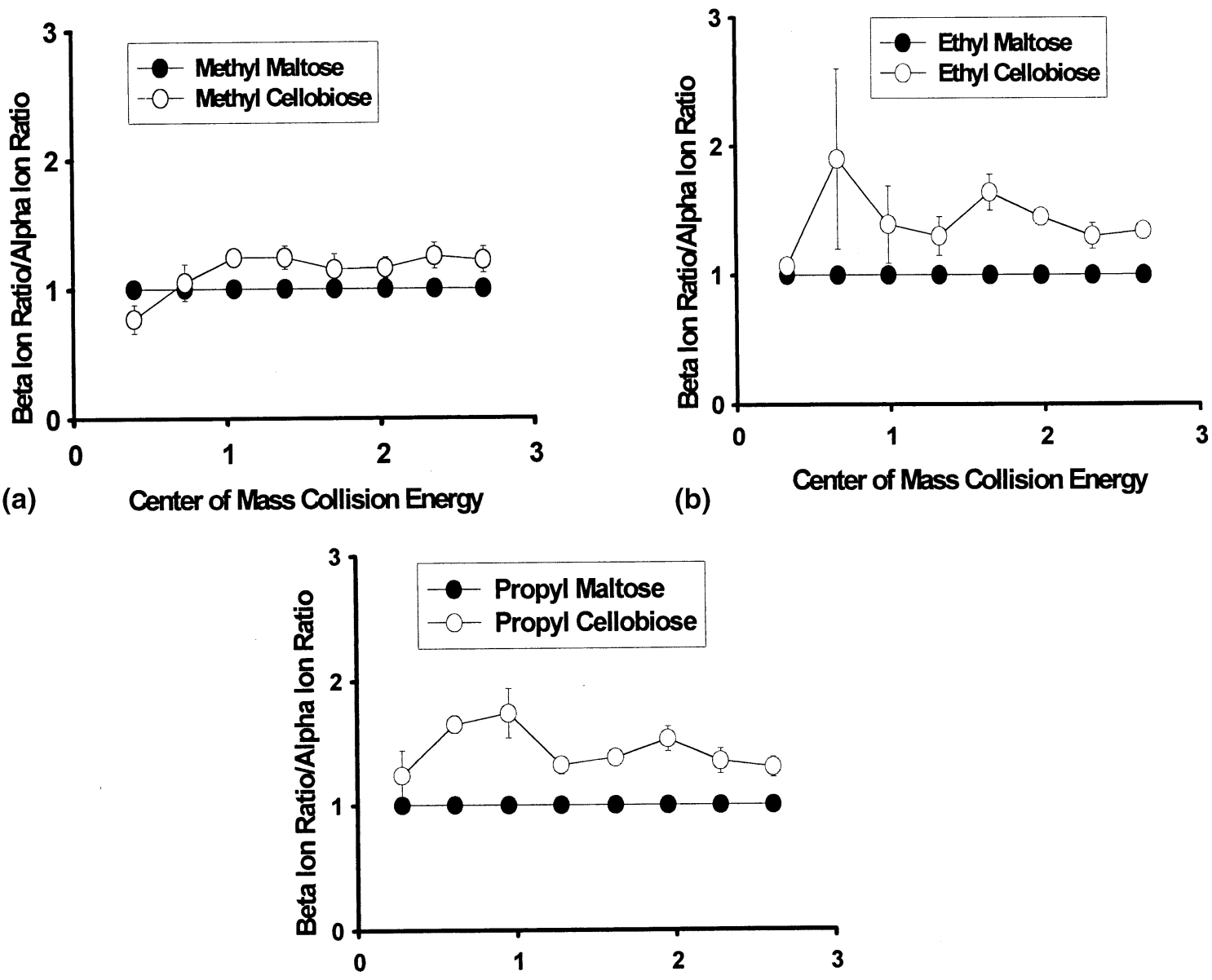

(c) Center of Mass Collision Energy
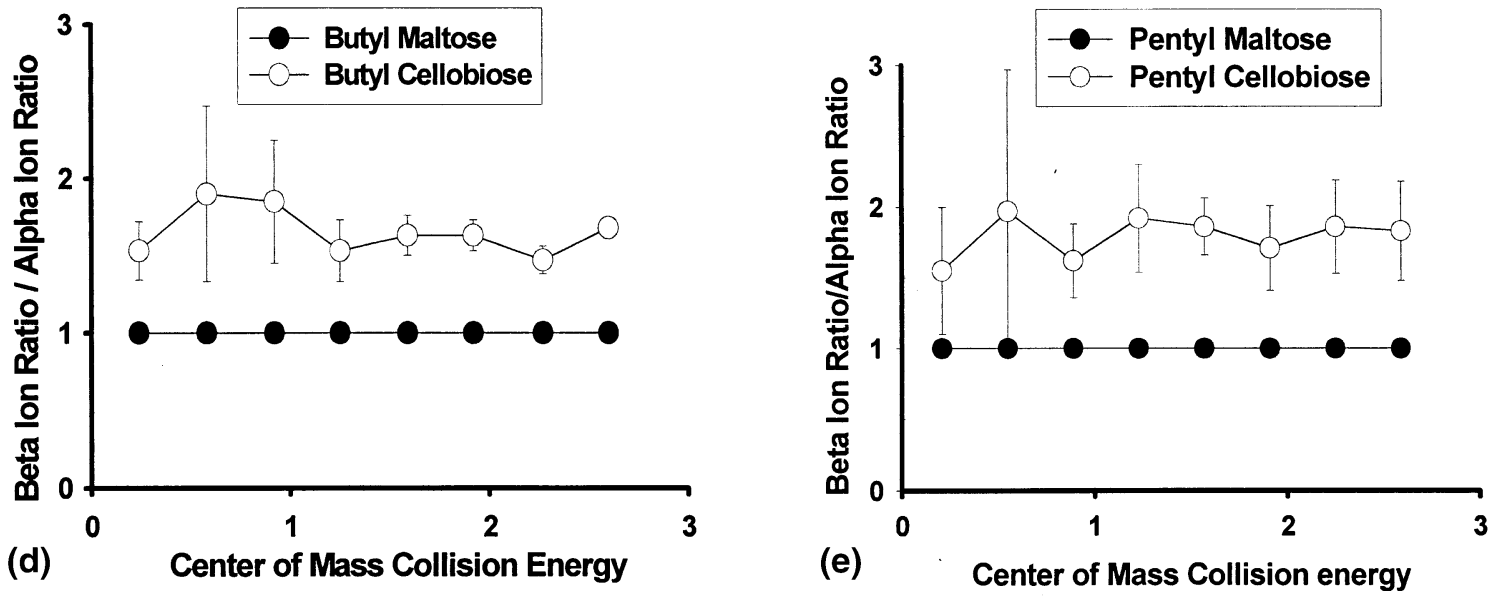

Figure 6. Anomeric configuration differentiation for $1 \rightarrow 4$ linked (a) permethylated, (b) perethylated, (c) perpropylated, derivatives. Anomeric configuration differentiation for $1 \rightarrow 4$ linked (d) perbutylated, (e) perpentylated, derivatives.

dissociation reflects differences in crowding between the $\alpha$ and $\beta$ bonds, as shown in this work, but discrimination between isomers is not as pronounced for the methylated derivative compared to higher derivatives. The barriers to internal rotation and thus the conformational freedom greatly depend on the location of sub- stituents in conformational space. Thus, as the size of the alkyl substituent is increased, especially to the largest alkyl group, pentyl, the probability of neighboring pentyl groups occupying a close conformational space is much higher than for methyl. Another aspect which is evident from the plots is that the $\beta$ isomer 


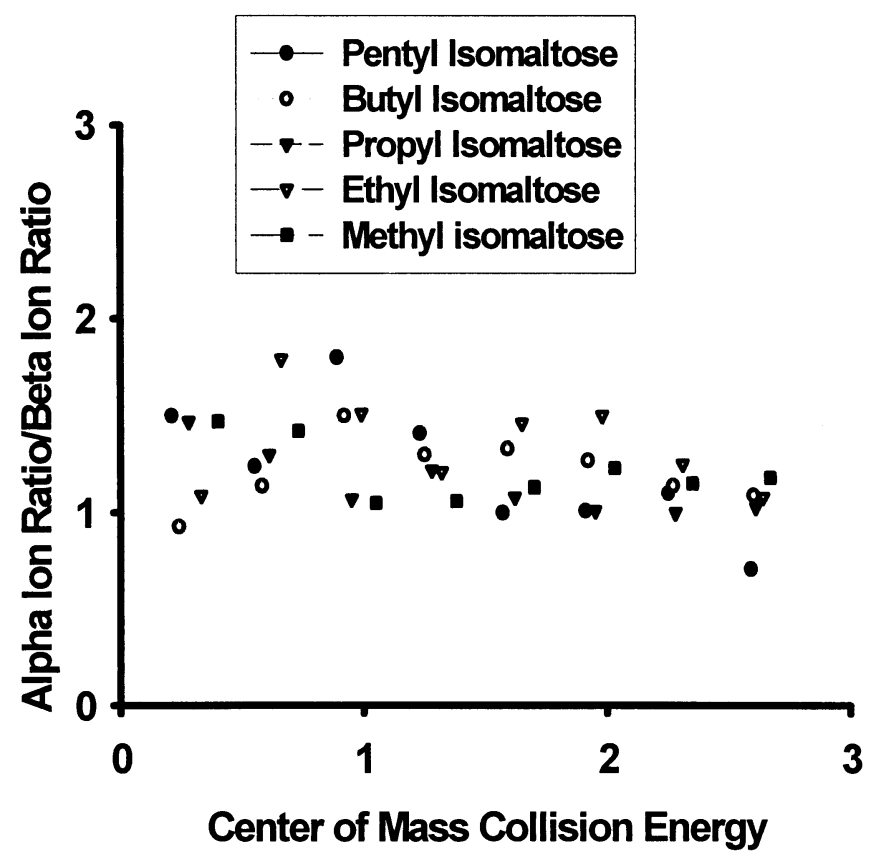

Figure 7. Consolidated plot for anomeric differentiation of $1 \rightarrow 6$ derivatives.

consistently has a higher daughter to parent ion ratio $(\mathrm{D} / \mathrm{P})$ than the corresponding $\alpha$ isomer in all five pairs of the $1 \rightarrow 4$ linkage isomer. A higher $\mathrm{D} / \mathrm{P}$ ion ratio means that the $\beta$ isomer is more readily cleaved. We had anticipated from Nilsson and coworkers' results with acetylated derivatives [51] that the $\beta$ isomer would be more resistant to decomposition than the corresponding $\alpha$ isomer since the $\beta$ bond is equatorial and should be more stable. Unexpectedly, a reverse trend is observed. One possibility is that the transition state could be governed by steric crowding of the substituents. Thus, permethylated cellobiose is more sterically restricted than permethylated maltose, which is corroborated by molecular modeling. Another possible explanation for this unusual phenomenon is a raising of the ground ionic state of the $\beta$ anomers thus lowering the activation barrier, enhancing the rate for $\beta$-glycosidic bond cleavage.Thus the $\beta$ isomer is kinetically more labile than the corresponding $\alpha$ isomer. It is reasonable to consider the activation energy because heterolytic bond dissociation energies are known to correlate well with activation energies as noted by Maccoll [71]. We can also attribute part of this trend to the anomeric effect which is a dipole-dipole repulsion between exocyclic and endocyclic $\mathrm{C}-\mathrm{O}$ bonds [72]. Briefly, in a $\beta$ isomer there is a dipole from the anomeric carbon to the sugar ring oxygen and to the glycosidic bond oxygen in the same direction. On the other hand, in the $\alpha$ isomer the dipole from the anomeric carbon to the sugar ring oxygen and to the glycosidic bond are in opposite directions. Thus, a greater dipole is present in the $\beta$ isomer causing it to have a higher ground state energy than the $\alpha$ isomer. Relieving this excess dipole moment is an energetic consequence causing the $\beta$ isomer to have a higher intrinsic chemical energy and thus fragment more easily in the collision chamber.

According to a second school of thought [73], it has been shown that the anomeric effect is due to stabilizing 2-electron delocalizations (hyperconjugation) from the oxygen lone pairs into adjacent polarized antiperiplanar $\sigma^{*} \mathrm{CO}$ bonds $\mathrm{n}(\mathrm{O}) \rightarrow \sigma^{*} \mathrm{CO}$ interactions. In this conformaton the $\sigma^{*} \mathrm{CO}$ orbital is anti to one of the lone pairs on the ring oxygen making it suitable for sigma electron delocalization. Sigma delocalization of electrons is known to stabilize a molecule and stabilize the charge on the molecule since sigma interactions are analogous to pi interactions as stated by Dewar [74]. Thus the rate of cleavage of the $\alpha$ isomer is reduced by through-bond interaction. In contrast, the $\beta$ isomer has the $\sigma^{*} \mathrm{CO}$ orbital gauche to the lone pairs on the ring oxygen, thus impeding sigma electron delocalization, hence charge stabilization, causing a faster rate of cleavage than the corresponding $\alpha$ isomer. Thus stereo-electronic factors, in addition to steric crowding of the freedom of motion of the glycosidic linkage, also play major roles in the differences in the rate of cleavage. The anomeric effect is known to have a $1-2 \mathrm{kcal} / \mathrm{mol}$ effect in bringing about an energy (enthalpy) difference between the $\alpha$ and $\beta$ isomers [51]. Such an enthalpy difference is sufficient to bring about a difference in the rate of cleavage of the glycosidic bond allowing the anomeric effect to play a major role in the rates of formation of ions related to cleavage of the glycosidic bond ion. Vanderkerk et al. [47] also attributed rates of formation of the glycosidic bond ion for the $\alpha$ and $\beta$ isomers of peracetylated xylobiosides ( $\alpha$ to $\beta$ ratio of 2.84) to an enthalpy difference of $4 \mathrm{~kJ} / \mathrm{mol}$. Thus, the $1 \rightarrow 4$ daughter ion 


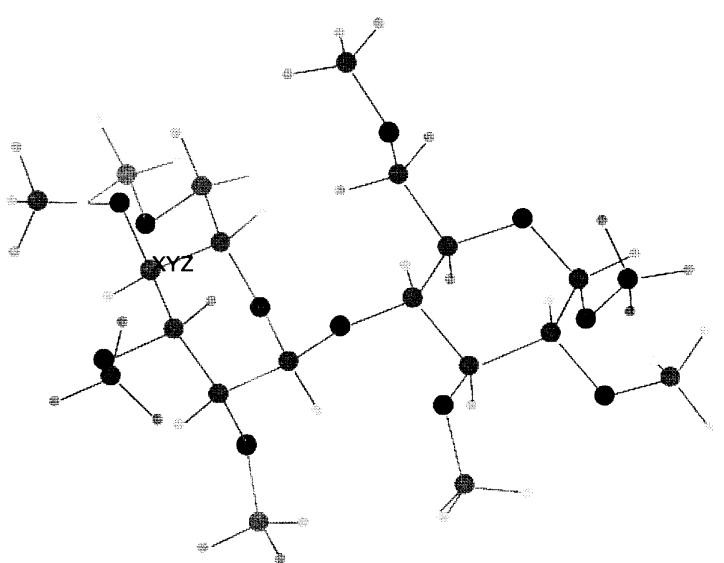

(a)

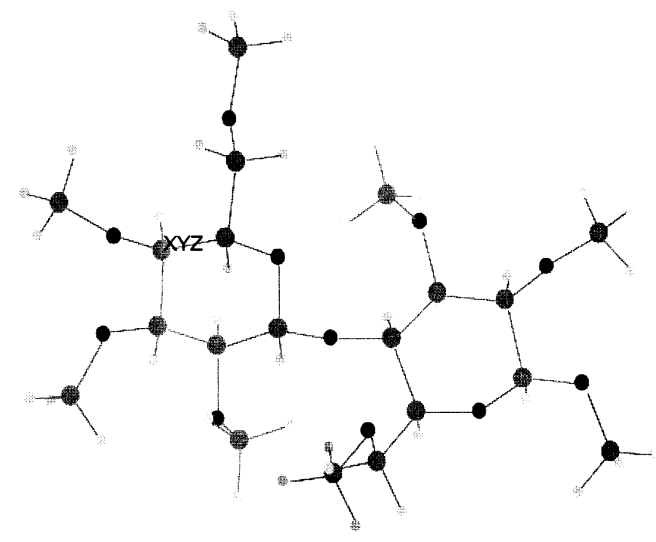

(b)

Figure 8. Minimum energy structures of permethylated (a) maltose, (b) cellobiose.

mass spectra of the $1 \rightarrow 4$ linkage isomers show ion abundances which are characteristic of each isomer.

$1 \rightarrow 6$ linkage pair. As a contrasting comparison, we chose the $1 \rightarrow 6$ derivative to show that little, if any contribution to the collision-induced cleavage is actually coming from the anomeric effect or other electronic parameters, but is largely due to the 3-D crowding of the glycosidic bond. Since the $1 \rightarrow 6$ linkages have three bonds to rotate about, the effects of alkyl substitution should be minimized, the results of which are shown in Figure 7. This is a consolidated plot of the $\alpha \mathrm{D} / \mathrm{P}$ ion ratio $/ \beta \mathrm{D} / \mathrm{P}$ ion ratio versus the center of mass collision energy, exhibiting little or no discrimination between the $\alpha$ and $\beta$ isomers for all the five pairs of derivatives. The $1 \rightarrow 6$ derivatives have much more rotational freedom of motion around the glycosidic bond than the corresponding $1 \rightarrow 4$ derivatives. This was validated by
Cummings and Carver [75], who found computationally that the three-bond $1 \rightarrow 6$ linkages are much more flexible than linkages involving only two bonds as in $1 \rightarrow 4$ linkages. In $1 \rightarrow 6$ linkages the three-bond rotation diminishes inter-residue interactions due to increased distance between the monosaccharide sugar rings. Dowd et al. [76] have demonstrated that both isomaltose and gentiobiose prefer extended orientations in which the two rings are placed trans relative to the O1-C6' bond. Therefore, both the isomers for all the different alkyl derivatives are able to rotationally diffuse the collision energy obtained during the collision event. Even though the alkyl incremented steric crowding around the glycosidic bond increases, conformational freedom of the three bond system precludes $1 \rightarrow 6$ anomeric discrimination. The $\alpha$ isomer appeared to exhibit a slight difference in $\mathrm{D} / \mathrm{P}$ ion ratio relative to the corresponding $\beta$, although whether this would hold up statistically is not certain. If the observation holds true, instead of the "anomeric" effect, this could be rationalized by invoking the exo-anomeric phenomenon $[77,78]$ in which the electron density of the lone pair of the exocyclic oxygen atom is transferred to the anti-bonding orbital of the endocyclic $\mathrm{C}-\mathrm{O}$ bond. Essentially this effect is maximized when the $p$ orbital for the unshared pair of electrons is anti-periplanar to the $\mathrm{C} 1$ ring oxygen. There are two conformations for the equatorially substituted anomer in comparison to only one for the axial substitution that are stabilized by the exo-anomeric effect. This causes a marginally lower $\mathrm{D} / \mathrm{P}$ ion ratio than that for the corresponding $\alpha$ anomer. Dowd et al. [76] have located minima having $\phi$ values between $-18^{\circ}$ and $-46^{\circ}$ for isomaltose in conformity with the exo-anomeric effect. This, in fact could slightly mitigate the effects shown for the $1 \rightarrow 4$ anomeric discrimination.

Lack of differentiation of $1 \rightarrow 4$ and $1 \rightarrow 6$ linkages in these studies. One of the conundrums of this study was that our previous work with methylated and underivatized trisaccharides had shown a more facile cleavage for the $1 \rightarrow 4$ than $1 \rightarrow 6$ linkages, while in these derivatized disaccharides, little difference was seen. Since the 6-linkage has a 3-bond rotation, and by the above references it was clear that more rotational freedom should make these bonds more stable to collision [1-3]. Thus the disaccharides are behaving differently than the trisaccharide models used before. We propose the following explanation: Even though the difference in the ease of fragmentation for the $1 \rightarrow 6$ derivatives is at least as high as that of the corresponding $1 \rightarrow 4$ derivatives, nonetheless, we consider that the torsional strain is still a predominant factor for promoting glycosidic bond cleavage. We take into consideration the possibility that the distribution of protonation sites is not equivalent for $1 \rightarrow 4$ and $1 \rightarrow 6$ isomeric pairs. Moreover, the distribution of protonation sites will change as the side chain length increases within either series. Because there is more space between sugar rings for the 


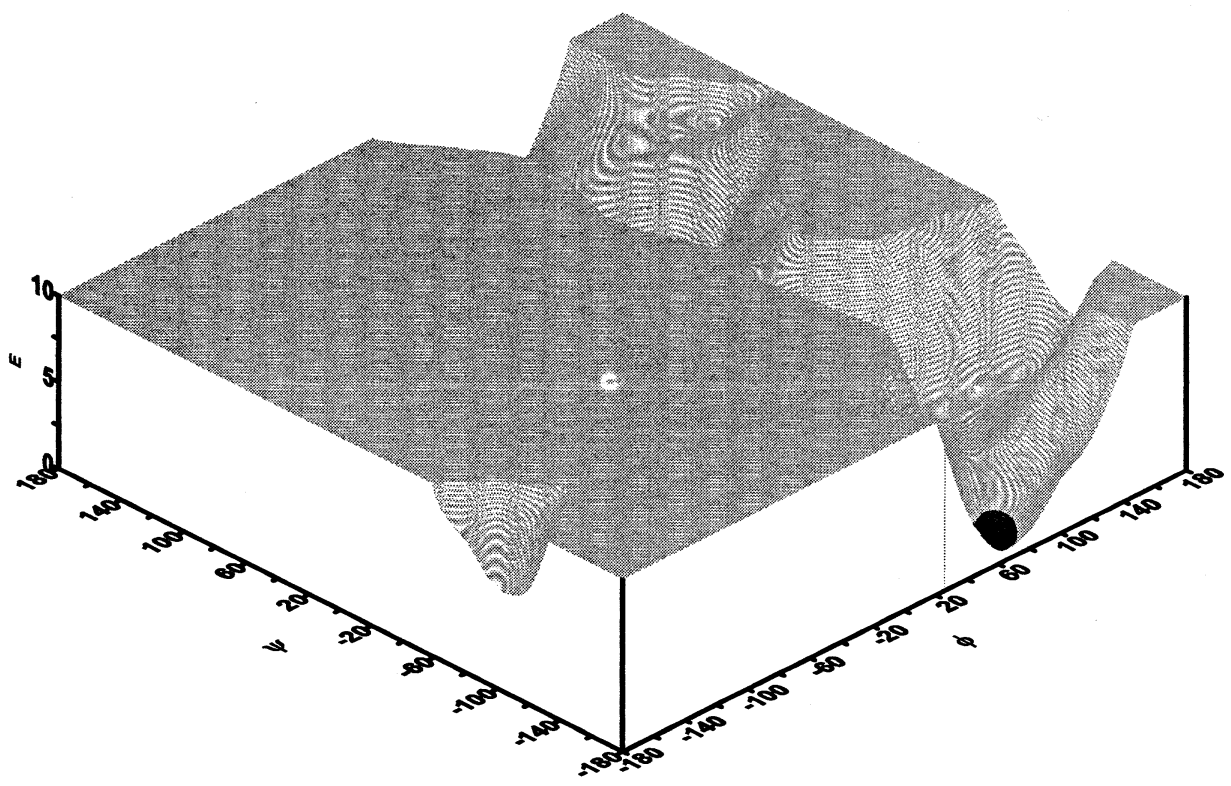

Figure 9. $\phi, \psi$. Energy plot of permethylated maltose.

$1 \rightarrow 6$ linkage, the propensity for protonation on the glycosidic linkage is less affected by increasing alkyl chain length for this series than for the $1 \rightarrow 4$ linkage series. When comparing the $1 \rightarrow 6$ series with the $1 \rightarrow 4$ series, the shifts in proton distributions with increasing alkyl chain length may thus offset to some degree the differences in amounts of increase in torsional strain. In other words, with increasing side chain length, relative torsional strain becomes more severe for the $1 \rightarrow 4$ series, while the relative propensity to protonate the glycosidic bond increases for the $1 \rightarrow 6$ series.

An additional factor that further complicates a direct comparison of $1 \rightarrow 4$ and $1 \rightarrow 6$ decompositions is that clearly alcohol loss is least facile from a 6-position alkoxy group where a primary carbocation must be formed upon decomposition. When a $1 \rightarrow 6$ disaccharide linkage exists, alcohol loss is more favorable because all alkoxy groups reside on secondary carbons.

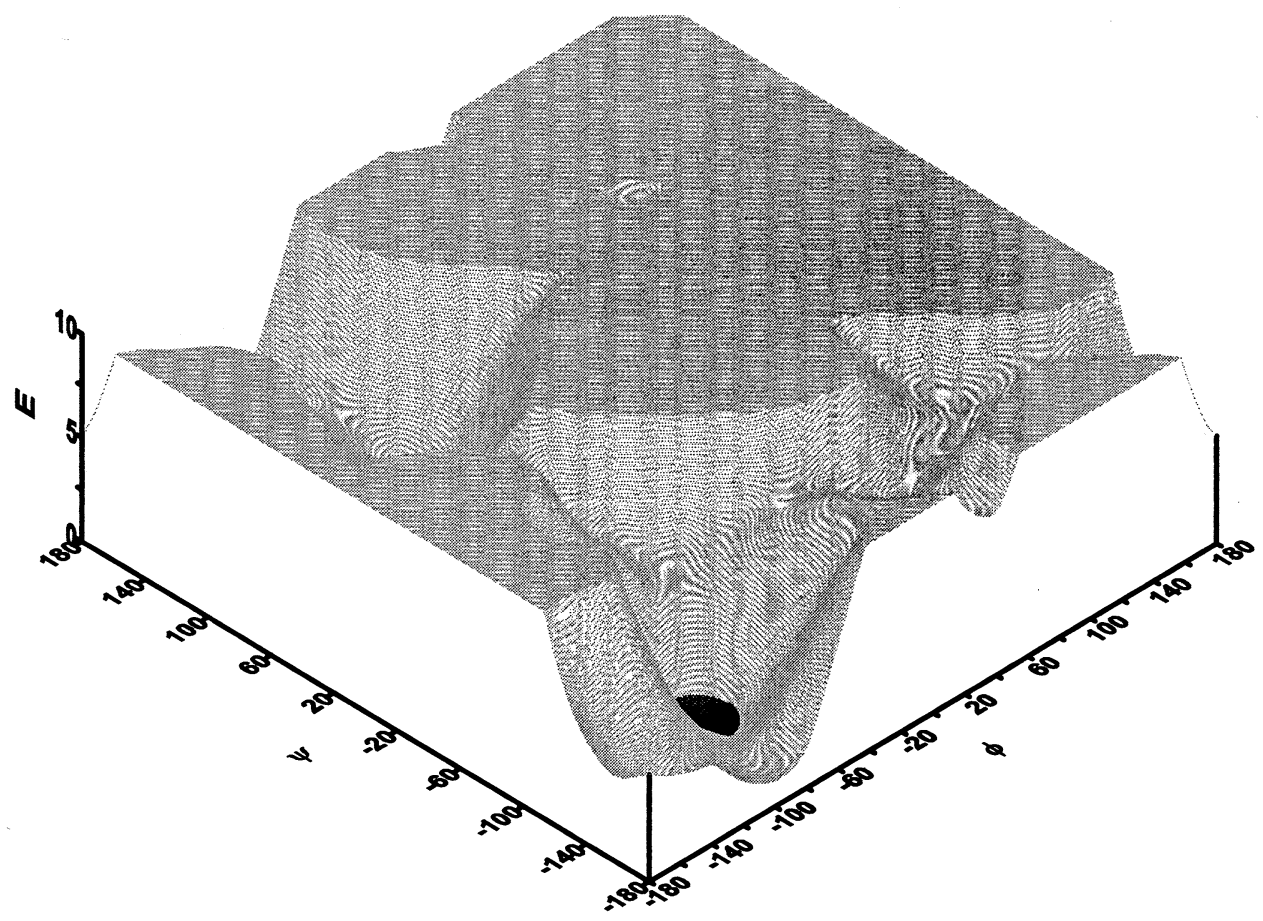

Figure 10. $\phi, \psi$. Energy plot of permethylated cellobiose. 
The relative ease of alcohol loss for the $1 \rightarrow 6$ linked series relative to the $1 \rightarrow 4$ series is evident upon inspection of Figures 2 and 3, and this separate route to decomposition contributes more heavily to the lowering of the parent ion signal for the $1 \rightarrow 6$ series. These two phenomena may contribute to the lessening of difference in ease of fragmentation for the $1 \rightarrow 4$ versus the $1 \rightarrow 6$ derivatives in the disaccharides studied here. In the trisaccharides studied previously [1-3], there may be some mitigation of these two effects by the large increase in total protonation sites made available by adding another sugar unit. However, these trisaccharides were only studied as the underivatized and methylated derivatives.

Modeling of $1 \rightarrow 4$ linkage anomers permethylated maltose and cellobiose. The fragmentation behavior of the disaccharides and dependence on freedom of motion reveal useful information concerning anomeric pair discrimination, further providing an opportunity to assess utility of molecular modeling programs for these systems. Computer modeling studies were undertaken for assessing the conformational freedom of motion around the glycosidic bond for the anomeric pair of permethylated maltose and cellobiose. Chem- $X$ was used to generate $\phi-\psi$ energy surfaces for $360^{\circ}$ rotations in $20^{\circ}$ increments about the $\phi$ and $\psi$ bonds. One hundred sixty-two starting structures were generated via a rules based search which enabled taking into account a limited number of starting structures. The (CID) experiments were carried out in vacuum, hence the modeling was carried out without solvation effects. Figure 8a and b shows the global minimum energy structures of permethylated maltose and cellobiose respectively optimized by MM3. Details of this modeling exercise are published elsewhere [79].

The modeling studies supported the fragmentation ratios and hence the degrees of freedom of motion observed under collision activation conditions in the triple quadrupole mass spectrometer for both the permethylated isomers. We had previously [1] assumed that bond scission for the carbon-oxygen bond of the glycosidic linkage would require on the order of $66 \mathrm{kcal}$ added energy and thus chose $55 \mathrm{kcal}$ as a benchmark. Figures 9 and 10 show 3-D energy wells of permethylated maltose and cellobiose, which are plots of $\phi, \psi$ and energy that are derived from MM3 calculations with a $10 \mathrm{kcal} \mathrm{mol}^{-1}$ cut off above the minima. The same benchmark of $55 \mathrm{kcal}$ was chosen with the difference that the 3-D wells were created from relative energy values, i.e., the difference between each energy value and the minimum energy value in the data file generated by MM3. We computed the volumes of the wells below $10 \mathrm{kcals}$ for both the isomers. The exact values of the volume are computed by the Surfer program. Permethylated cellobiose exhibited a smaller volume of $1.059 \mathrm{e}^{6} \mathrm{deg}^{2} \times \mathrm{kcal}$ than its corresponding isomer permethylated maltose which had a volume of $1.132 \mathrm{e}^{6}$ $\mathrm{deg}^{2} \times \mathrm{kcal}$. Thus, setting the volume of the well for permethylated cellobiose as 1.0, permethylated maltose gave a volume of 1.07. We claimed that volumes of the energy wells correspond to the conformational freedom of motion of the two monosaccharide sugar rings about the glycosidic bond [1] with the rate of dissociation having an inverse relationship to volume. The freedom of motion is correlated with the ability of the molecule to dissipate the energy obtained in the collision event. Thus permethylated maltose, which has a greater volume, will dissipate the added collision energy among its internal modes more easily than permethylated cellobiose and thus exhibits a greater stability in the mass spectrometer. Values of the volumes are similar, which corroborates with the small difference in fragmentation ratios obtained between the two isomers is due to a very similar degree of motional freedom. Comparison of volumes of the wells below a relative energy of 10 kcals gives an estimate of the relative stability of the glycosidic bond to energy imparted to the molecule in the collision event. However, electronic considerations, as described above, as well as results from previous work [1-3] suggest that disaccharide models may give different results than models with higher oligomers.

\section{Conclusions}

Electrospray tandem mass spectrometry is a useful analytical approach for depicting bond stability to collision-induced activation of derivatized disaccharides. The CID fragmentation patterns gave ions corresponding exclusively to cleavage of the glycosidic bond showing increased frangibility of the glycosidic bond upon incremented alkyl substitution, and incidentally giving information on the anomericity of the glycosidic bond for the ring-linked derivatives. A comparison of the alkylated derivatives for a particular isomer revealed a smooth trend in incremented bond frangibility from methyl to pentyl for $1 \rightarrow 4$ and $1 \rightarrow 6$ derivatives with the pentylated derivative being the least stable to collision in the mass spectrometer. This methodology also showed differentiation of $1 \rightarrow 4$ linked anomeric pairs but not $1 \rightarrow 6$. The spectra obtained are strongly dependent on the freedom of rotation of the monosaccharide rings about the glycosidic bond. Although further studies of model systems will be required before this approach can be routinely used, we believe that this methodology may become useful in high sensitivity discernment of structural problems in carbohydrates.

\section{Acknowledgments}

This work was partially supported by NSF CHE-9981948 and by funds from the Louisiana Educational Quality Support Fund (LEQSF). 


\section{References}

1. Laine, R. A.; Pamidimukkala, K.; French, A. D.; Hall, R. W.; Abbas, S. A.; Jain, R. K.; Matta, K. L. J. Am. Chem. Soc. 1988, 110, 6931-6938.

2. Laine, R. A.; Yoon, E.; Mahier, T. J.; Abbas, S. A.; de Lappe, B.; Jain, R. K.; Matta, K. L. Biol. Mass Spectrom. 1991, 20, 505-514.

3. Yoon, E.; Laine, R. A. Biol. Mass Spectrom. 1992, 21, 479-485.

4. Domon, B.; Mueller, D. R.; Richter, W. J. Org. Mass Spectrom. 1989, 24, 357-359.

5. Domon, B.; Mueller, D. R.; Richter, W. J. Int. J. Mass Spectrom. Ion Processes 1990, 100, 301-311.

6. Albersheim, P.; Nevins, D. J.; English, P. D.; Karr, A. Carb. Res. 1967, 5, 340-345.

7. Sweely, C. C.; Bentley, R.; Makita, M.; Wells, W. W. J. Am. Chem. Soc. 1963, 85, 2497-2507.

8. Sawardekar, J. S.; Sloneker, J. H.; Jeanes, A. R. Anal. Chem. 1965, 37, 1602-1604.

9. Golovkina, L. S.; Chizhov, O. S.; Wultson, U. S. Bull. Acad. Sci. U.S.S.R. 1966, 11, 1853-1863.

10. Poulter, L.; Earnest, J. P.; Stroud, R. M.; Burlingame, A. L. Proc. Natl. Acad. Sci. U.S.A. 1989, 86, 6645-6650.

11. Lindberg, B.; Longren, J. Methods Enzymol. 1978, 50, 3-33.

12. Svensson, S. Methods Enzymol. 1978, 50, 33-38.

13. Valent, B. S.; Darvill, A. G.; Mcneil, M.; Robertson, B. K.; Albersheim, P. Carb. Res. 1980, 79, 165-192.

14. Samuelson, B. E.; Pimlott, W.; Karlsson, K. A. Methods Enzymol. 1990, 193, 623-646.

15. Hellerqvist, C. G. Methods Enzymol. 1990, 193, 554-573.

16. Gray, G. R. Methods Enzymol. 1990, 193, 573-587.

17. Mulroney, B.; Traeger, J. C.; Stone, B. A. J. Mass Spectrom. 1995, 30, 1277-1283.

18. Smith, G.; Leary, J. A. J. Am. Soc. Mass Spectrom. 1996, 7, 953-957.

19. Li, D. T.; Her, G. R. J. Mass Spectrom. 1998, 33, 644-652.

20. Gaucher, S. P.; Leary, J. A. J. Am. Soc. Mass Spectrom. 1999, 10, 269-272.

21. Dwek, R. A. Chem. Rev. (Washington DC) 1996, 96, 683-720.

22. Varki, A.; Cummings, R.; Esko, J.; Freeze, H.; Hart, G.; Marth, J. Essentials of Glycobiology. Cold Spring Harbor Laboratory Press, 1999.

23. Laine, R. A. Pure Appl. Chem. 1997, 69, 1867-1873.

24. Laine, R. A. Glycobiology 1994, 6, 759-767.

25. Edge, C. J.; Rademacher, T. W.; Wormald, M. R.; Parekh, R. B.; Butters, T. D.; Wing, D. R.; Dwek, R. A. Proc. Natl. Acad. Sci. U.S.A. 1992, 89, 6338-6342.

26. Matheson, N. K.; Mcleary, B. V. In The Polysaccharides; Aspinall, G., Ed.; Academic Press: New York, 1985; pp 1-31 Chap III.

27. Lee, E. Y. C.; Whelan, W. J. Arch. Biochem. Biophys. 1966, 116, 162-167.

28. Li, Y. T.; Li,, S. C. In Methods in Carbohydrate Chemistry. Whistler, R. L.; Bemiller, J. N., Eds.; Academic Press: New York, 1976; pp 221-225 Chap VII.

29. Aspinall, G. O. Tech. Chem. 1973, 4, 379-450.

30. Barry, V. C.; McCormick, J. E.; Mitchell, P. W. D. J. Chem. Soc. 1954, 3692-3696.

31. Goldstein, I. J.; Hay, G. W.; Lewis, B. A.; Smith, F. In Methods in Carbohydrate Chemistry. Whistler, R. L., Ed.; Academic Press: New York, 1965; pp 361-370 Chap V.

32. Angel, A. S.; Nilsson, B. Methods Enzymol. 1990, 193, 587-607.

33. Hakomori, S. J. Biochem. (Tokyo) 1964, 55, 205-208.

34. Bjorndal, H.; Lindberg, B.; Svensson, S. Acta Chem. Scan. 1967, 21, 1801-1804.

35. Bjorndal, H.; Lindberg, B.; Svensson, S. Carb. Res. 1967, 5, 433-440.
36. Hellerqvist, C. G.; Lindberg, B.; Svensson, S.; Holme, T.; Lindberg, A. A. Carb. Res. 1968, 8, 43-55.

37. Bjorndal, H.; Hellerqvist, C. G.; Lindberg, B.; Svensson, S. Angew Chem. 1979, 82, 643-650.

38. Lindberg, B.; Lonngren, J. In Methods in Carbohydrate Chemistry. Whistler, R. L.; Bemiller, J. N., Eds.; Academic Press: New York, 1976; pp 142-148 Chap VII.

39. Angyal, S. J.; James, K. Aust. J. Chem. 1970, 23, 1209-1221.

40. Halbeek, H. V. Curr. Opin. Struct. Biol. 1994, 4, 697-709.

41. Bush, C. A. Curr. Opin. Struct. Biol. 1992, 2, 655-660.

42. Carver, J. Curr. Opin. Struct. Biol. 1991, 1, 716-720.

43. Bush, C. A. Biophys. J. 1994, 66, 1267-1268.

44. Richter, W. J.; Blum, W.; Schlunegger, U. P.; Senn, M. In Mclafferty, F. W. ed.Tandem Mass Spectrometry, Wiley: New York, 1983; pp 417-434.

45. Mueller, D. R.; Domon, B.; Blum, W.; Raschdorf, F.; Richter, W. J. Biomed. Environ. Mass Spectrom. 1988, 15, 441-446.

46. Khoo, K. H.; Dell, A. Glycobiology 1990, 1, 83-91.

47. Van der Kerk, S. M.; Blok-Tip, L. Kerk-; van Hoof, A. V.; Heerma, W.; Haverkamp, J. Int. J. Mass Spectrom. Ion Processes 1994, 134, 41-54.

48. Brakta, M.; Chaguir, B.; Sinou, D.; Banoub, J.; Becchi, M. Org. Mass Spectrom. 1992, 27, 331-339.

49. Brakta, M.; Sinou, D. Banoub, J;; Becchi, M. Org. Mass Spectrom. 1992, 27, 621-624.

50. Dallinga, J. W.; Heerma, W. Biol. Mass Spectrom. 1991, 20, 215-231.

51. Fangmark, I.; Jansson, A.; Nilsson, B. Anal. Chem. 1999, 71, 1105-1110.

52. Mulroney, B.; Peel, J. B.; Traeger, J. C. J. Mass Spectrom. 1999, 34, 856-871.

53. Williams, D.; Young, M. K. Rapid Commun. Mass Spectrom. 2000, 14, 2083-2091.

54. Burlingame, A. L.; Boyd, R. K.; Gaskell, S. J. Anal. Chem. 1994, 66, 634R-683R

55. Reinhold, V. N.; Reinhold, B. B.; Costello, C. E. Anal. Chem. 1995, 67, 1772-1784.

56. Ohasi, Y. In Electrospray Ionization Mass Spectrometry. Cole, R. B., Ed.; John Wiley and Sons: New York, 1997; pp 459-498.

57. Cicanu, I.; Kerek, F. Carb. Res. 1984, 113, 157-162.

58. Allinger, N. L.; Rahman, M.; Lii, J. H. J. Am. Chem. Soc. 1990, $112,8293-8307$.

59. Viseux, N.; de Hoffmann, E.; Domon, B. Anal. Chem. 1997, 69 , 3193-3198.

60. Domon, B.; Costello, C. E. Glycoconj. J. 1988, 5, 397-409.

61. Viseux, N; de Hoffman, E.; Domon, B. Anal. Chem. 1998, 70, 4951-4959.

62. Kochetkov, N. K.; Chizhov, O. S. Adv. Carbohydr. Chem. 1966, $21,39$.

63. Dell, A. Adv. Carb. Chem. Biochem. 1987, 45, 19-72.

64. Hayes, R. N.; Gross, M. L. Methods Enzymol. 1990, 193, 237-263.

65. Bernstein, R. B.; Levine, R. D. Molecular Reaction Dynamics. Clarendon Press: Oxford, 1974.

66. Nystrom, J. A.; Bursey, M. M.; Hass, J. R. Int. J. Mass Spectrom. Ion Processes 1983/1984, 55, 263-274.

67. Schey, K. L.; Kenttamaa, H. I.; Wysocki, V. I.; Cooks, R. G. Int. J. Mass Spectrom. Ion Processes 1983, 90, 71-83.

68. Holmes, J. L. Org. Mass Spectrom. 1985, 20, 169-183.

69. Cole, R. B.; Tabet, J. C.; Salles, C.; Jallageas, J. C.; Crouzet, J. Rapid Commun. Mass Spectrom. 1989, 3, 59-61.

70. Blake, J. F.; Jorgenson, W. L. J. Org. Chem. 1991, 56, 6052-6059.

71. MacColl, A. Chem. Rev. 1969, 69, 33-60.

72. Lemieux, R. U.; Chu, N. J. Am. Chem. Soc. (abstract of papers) $1958,133,31 \mathrm{~N}$ 
73. Petillo, P. A.; Lerner, L. E. Origin and Quantitative Modeling of Anomeric Effect; Chap IX.

74. Dewar, M. J. S. J. Am. Chem. Soc. 1984, 106, 669-682.

75. Cummings, D.; Carver, J. Biochemistry 1987, 26, 6676-6683.

76. Dowd, M. K.; Reilly, P. J.; French, A. D. Bipolymers 1994, 34, 625-638.
77. Lemieux, R. U.; Pavia, A. A.; Martin, J. C.; Watanabe, K. A. Can. J. Chem. 1969, 47, 4427-4439.

78. Lemieux, R. U.; Pavia, A. A.; Martin, J. C. Can. J. Chem. 1987, 65, 213-223.

79. Mendonca, S.; Johnson, G. P.; French, A. D.; Laine, R. A. J. Phys. Chem. A 2002, 106, 4115-4124. 